\title{
新型多功能无机/有机复合薄膜的制备及电化学性能研究
}

\author{
陈 钧 ${ }^{1}$, 马培华 ${ }^{2}$, 张 诚 ${ }^{1}$, 劳伦 . 鲁尔曼 ${ }^{3}$, 吕耀康 ${ }^{1}$
}

(1. 浙江工业大学 化学工程学院, 杭州 310014；2. 绍兴金冶环保科技有限公司, 绍兴 312073; 3. 斯特拉斯堡大学 化学研究所, 斯特拉斯堡 67081, 法国)

摘 要: 将铈钛氧簇 $\left[\mathrm{Ti}_{8} \mathrm{O}_{7}(\mathrm{HOEt})(\mathrm{OEt})_{21} \mathrm{Ce}\right]$ 和 PEDOT 低聚物的混合溶液通过滴涂-二次聚合成膜制得一种表面具有特 殊纳米沟壑结构的无机/有机复合薄膜 PEDOT:Ce@ $\mathrm{TiO}_{2}$ 。PEDOT:Ce@ $\mathrm{TiO}_{2}$ 具有很强的疏水性和对乙腈溶液较好的润 湿性, 能用作阴极电致变色材料和超级电容器电极材料。PEDOT:Ce@ $\mathrm{TiO}_{2}$ 展现出较 PEDOT 薄膜更优良的电化学性 能, 在电流密度为 $1 \mathrm{~A} / \mathrm{g}$ 时, PEDOT:Ce $@ \mathrm{TiO}_{2}$ 的质量比电容为 $71.2 \mathrm{~F} / \mathrm{g}$, 是相同条件下 PEDOT 薄膜的质量比电容的 1.7 倍。采用 PEDOT:Ce@ $\mathrm{TiO}_{2}$ 进一步组装了全固态电致变色超级电容器原型器件, 当充电完成时器件的变色区域呈现墨 绿色, 当放电完成时器件的变色区域呈现亮黄色。

关 键 词: 聚 3,4-乙烯二氧噻吩; 钛氧族; 疏水性; 电致变色薄膜; 超级电容器

中图分类号: 0614 文献标识码: A

\section{Preparation and Electrochemical Property of New Multifunctional Inorganic/Organic Composite Film}

\author{
CHEN Jun ${ }^{1}$, MA Pei-Hua ${ }^{2}$, ZHANG Cheng ${ }^{1}$, Laurent RUHLMANN ${ }^{3}$, LYU Yao-Kang ${ }^{1}$ \\ (1. School of Chemical Engineering, Zhejiang University of Technology, Hangzhou 310014, China; 2. Shaoxing Jinye Envi- \\ ronmental Protection Technology Co., Ltd., Shaoxing 312073, China; 3. Institut de Chimie, Université de Strasbourg, Stras- \\ bourg 67081, France)
}

\begin{abstract}
A new inorganic/organic composite film PEDOT:Ce@ $\mathrm{TiO}_{2}$ was prepared through drop casting-secondary polymerization method using the mixture solution of PEDOT oligomer and cerium-containing polyoxotitanate cage $\left[\mathrm{Ti}_{8} \mathrm{O}_{7}(\mathrm{HOEt})(\mathrm{OEt})_{21} \mathrm{Ce}\right]$. PEDOT:Ce@ $\mathrm{TiO}_{2}$ exhibits a special nanostructure with gully-like rough surface, which shows strong hydrophobicity while good wettability to acetonitrile solution. PEDOT:Ce@ $\mathrm{TiO}_{2}$ can be used as cathodically electrochromic film and supercapacitor electrode material. The mass specific capacitance of PEDOT:Ce@TiO is $71.2 \mathrm{~F} / \mathrm{g}$ at a current density of $1 \mathrm{~A} / \mathrm{g}$, which is 1.7 times higher than that of the PEDOT film. An all-solid-state electrochromic supercapacitor prototype device was further assembled using PEDOT:Ce@ $\mathrm{TiO}_{2}$. With charging completed the electrochromic area of this device shows dark green, and it turns bright yellow with discharging completed.
\end{abstract}

Key words: poly 3,4-ethylenedioxythiophene; polyoxotitanate; hydrophobicity; electrochromic film; supercapacitor

超级电容器是一种新型高效的电化学储能装置, 其电化学性能主要取决于电极材料的组成和结构 ${ }^{[1-7]}$ 。
近年来，随着可穿戴、便携式和智能化电子产品的 迅猛发展，对储能元件的智能化、集成化和多功能

收稿日期：2019-04-15; 收到修改稿日期：2019-07-12

基金项目: 浙江省自然科学基金(LY19B010003); 国家自然科学基金(21501148)

Zhejiang Provincial Natural Science Foundation (LY19B010003); National Natural Science Foundation of China (21501148)

作者简介：陈 钧(1992-)，男，硕士研究生. E-mail: 1192759665@qq.com

CHEN Jun(1992-), male, Master candidate. E-mail: 119275966@qq.com

通讯作者: 吕耀康, 校聘副研究员. E-mail: yaokanglv@zjut.edu.cn

LYU Yao-Kang, associate professor. E-mail: yaokanglv@zjut.edu.cn 
化提出了更加迫切的要求, 新型超级电容器电极材

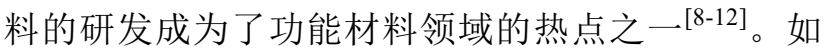
果超级电容器能及时感应到储存电能的变化, 并以 一种明显的方式向使用者展示这一变化, 使用者就 可以在电子设备停止工作之前, 快速确定能量消耗 状况并进行相应操作 ${ }^{[13-14]}$ 。研究表明, 许多共轭聚 合物和过渡金属氧化物材料既有较大的质量比电容, 也有较好的电致变色性能 ${ }^{[15-20]}$, 将同时具有储电能 力和电致变色性能的多功能材料应用在超级电容器 中, 可研制在充放电过程中提供根据储电状态呈现 不同颜色的电致变色超级电容器, 把能量存储和电 致变色功能集成在一个智能设备中 ${ }^{[21-23]}$ 。

聚(3,4-乙烯二氧噻吩), 简称 PEDOT, 是一种 分子结构简单、能隙小、电导率高的共轭聚合物, 能 在发生氧化还原反应的同时发生明显的颜色变化, 作为电致变色器件和超级电容器的电极材料得到了 广泛研究 ${ }^{[24-26]}$ 。纯 PEDOT 聚合度高, 溶解性差, 加 工和电化学循环稳定性问题限制了其应用范围 ${ }^{[27]}$ 。 比利时爱克发等公司将水溶性聚合物电解质聚苯乙 烯磺酸(PSS)与 PEDOT 复合, 解决了 PEDOT 的加工 问题, 生产的 PEDOT:PSS 复合材料可以作为导电 油墨等产品的活性成分; LIU 等 ${ }^{[28]}$ 将 PEDOT:PSS 进 一步与粒径为 $15 \mathrm{~nm}$ 的纳米 $\mathrm{TiO}_{2}$ 复合, 制备了 PEDOT:PSS- $\mathrm{TiO}_{2}$ 复合材料及基于该材料的电致变 色器件, 在 PEDOT:PSS- $\mathrm{TiO}_{2}$ 中电子和空穴的有效 分离使该材料的稳定性与 PEDOT:PSS 相比有了大 幅提高。钛氧簇 ${ }^{[29-31]}$ 不仅具有与纳米 $\mathrm{TiO}_{2}$ 相似的内 核结构和光电性质, 而且分子结构可控, 表面基团 易于修饰, 在功能材料领域展现出广阔的应用前 景。铈钛氧族 $\left[\mathrm{Ti}_{8} \mathrm{O}_{7}(\mathrm{HOEt})(\mathrm{OEt})_{21} \mathrm{Ce}\right]$ 具有直径约 $1 \mathrm{~nm}$ 的内核 $\mathrm{CeTi}_{8} \mathrm{O}_{29}$; 溶于二氯甲烷等有机溶剂, 可以通过水解去除 $\left[\mathrm{Ti}_{8} \mathrm{O}_{7}(\mathrm{HOEt})(\mathrm{OEt})_{21} \mathrm{Ce}\right]$ 表面的乙
氧基团, 得到 $\mathrm{Ce}: \mathrm{Ti}$ 原子比为 $1: 8$ 的 $\mathrm{Ce}(\mathrm{III})$ 掺杂 $\mathrm{TiO}_{2}\left(\mathrm{Ce} @ \mathrm{TiO}_{2}\right)^{[32]}$ 。

本论文尝试将铈钛氧簇 $\left[\mathrm{Ti}_{8} \mathrm{O}_{7}(\mathrm{HOEt})(\mathrm{OEt})_{21} \mathrm{Ce}\right]$ 和 PEDOT 结合制备新型的无机/有机复合薄膜新材 料, 研究这种新材料的电化学性能, 并对它的应用 前景作了初步探索。

\section{1 实验方法}

\section{1 薄膜的制备}

如图 1 所示, 通过化学氧化法制备PEDOT 低聚 物溶液(图 S2(a)), 按参考文献[32]的方法合成铈钛 氧族 $\left[\mathrm{Ti}_{8} \mathrm{O}_{7}(\mathrm{HOEt})(\mathrm{OEt}){ }_{21} \mathrm{Ce}\right]$, 溶于二氯甲烷配制 $0.03 \mathrm{~mol} / \mathrm{L}$ 的铈钛氧簇溶液 (图 S2(b))。将配制的铈 钛氧族溶液与 PEDOT 低聚物溶液混合后(图 S2(c)) 通过滴涂法涂布在氧化铟锡 (ITO) 玻璃表面, 在 $60{ }^{\circ} \mathrm{C}$ 下二次聚合并洗涤、烘干，制得 PEDOT:Ce@ $\mathrm{TiO}_{2}$ 复合薄膜(图 S2(e)); 采用相同的 滴涂法制得未复合的 PEDOT 薄膜作为比较(图 S2(d)) (制备步骤详见补充材料 S2 S5)。

\section{2 薄膜结构表征与性能测试}

红外光谱(IR)采用美国赛默飞尼高力 6700 型傅 里叶变换红外光谱仪测定; 紫外-可见光谱图 (UV-Vis)采集于日本岛津 UV-1800 紫外-可见分光 光度计; $X$ 射线衍射(XRD)光谱数据在荷兰帕纳科 公司的 X'Pert Pro 高分辨 X 射线衍射仪上获得; 扫 描电子显微镜(SEM)照片和 $X$ 射线能谱(EDS)分析 采集于日本日立 S-4800 型场发射扫描电子显微镜; 原子力显微镜(AFM) 分析数据采集于美国布鲁 Dimension 原子力显微镜; 相对分子量测试由搭载 Water 2487 双波长紫外一可见光检测器和 Waters 2414 示差折光检测器的 Waters 1525/2414 凝胶渗透

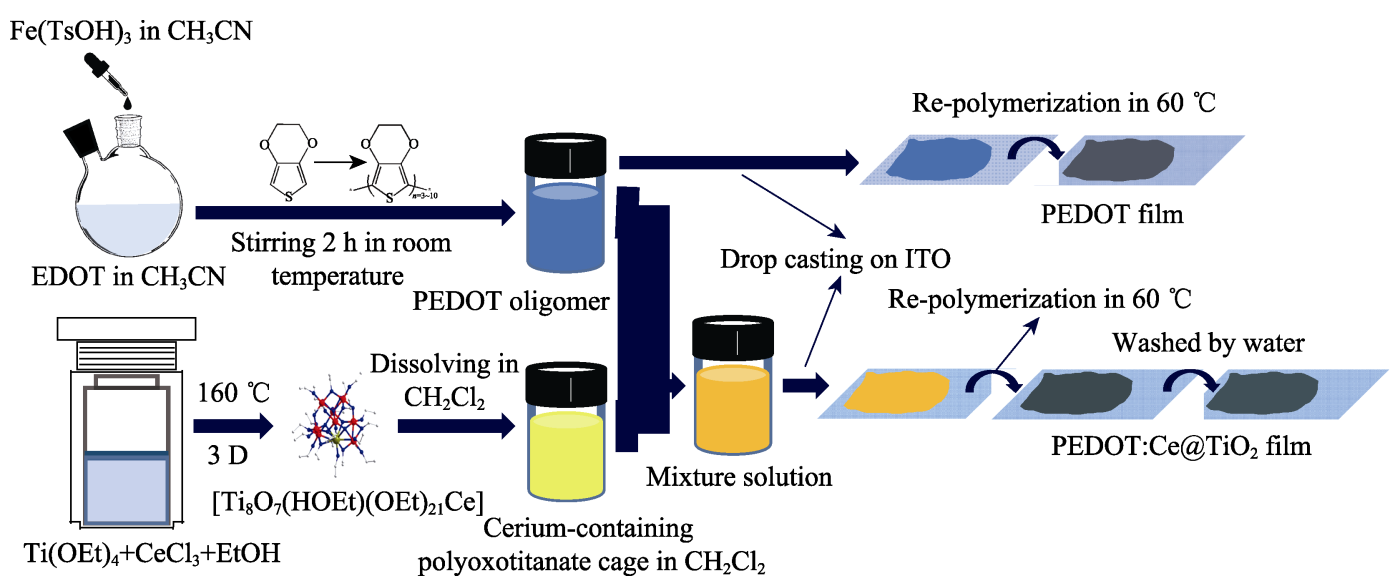

图 1 PEDOT:Ce@ $\mathrm{TiO}_{2}$ 薄膜的实验制备流程图

Fig. 1 Schematic illustration of preparation of PEDOT:Ce@ $\mathrm{TiO}_{2}$ film 
色谱仪(GPC)测得; 材料表面润湿性能采用测量水 滴与材料表面的接触角获得, 利用德国 Dataphysics 公司 dataphysics OCA50 全自动接触角测量仪进行 测试。光谱电化学测试采用日本岛津 UV-1800 紫外 -可见分光光度计和上海辰华 $\mathrm{CHI} 660 \mathrm{E}$ 电化学工作 站联用技术; 循环伏安 $(\mathrm{CV})$ 、恒流充放电 $(\mathrm{GCD})$ 和 交流阻抗(EIS)分析采用 CHI660E 电化学工作站测 定(测试方法详见补充材料 S8)。

\section{2 结果与讨论}

\section{1 薄膜的结构表征}

采用 GPC 表征 PEDOT 低聚物溶液的相对分子 质量及其分布(图 S1)的结果表明, 在 PEDOT 低聚 物溶液中, 聚合度主要分布在 3 10 之间。然而由于 在烘干成膜过程中发生了二次聚合反应，制备的 PEDOT 薄膜和 PEDOT:Ce $@ \mathrm{TiO}_{2}$ 复合薄膜具有更高 的聚合度, 所以不能溶解在乙腈、二氯甲烷等有机 溶剂中。PEDOT:Ce@ $\mathrm{TiO}_{2}$ 和 PEDOT 具有相似的 FT-IR 光谱图(图 S3)。如图 S4 所示, PEDOT 的粉末 XRD 图谱在 $2 \theta$ 为 $27^{\circ}$ 附近出现了一个强的 PEDOT 特征衍射峰 ${ }^{[33]}$; 而 PEDOT: $\mathrm{Ce} @ \mathrm{TiO}_{2}$ 的粉末 XRD 图 谱在衍射角 $2 \theta$ 为 $15^{\circ} \sim 35^{\circ}$ 之间出现了一个馒头形的 弥散衍射峰, 表明其具有与 $\mathrm{Ce} @ \mathrm{TiO}_{2}$ 粉末类似的无 定形结构 ${ }^{[32]}$ 。

图 2(a) 是 PEDOT 薄膜的 SEM 照片, 可见 PEDOT 薄膜较平整, 而 PEDOT:Ce@ $\mathrm{TiO}_{2}$ 表面呈现
出特殊的纳米沟壑状微观形貌(图 2(d))。EDS 能谱 分析表明(补充材料 S7), 在 PEDOT:Ce@ $\mathrm{TiO}_{2}$ 薄膜 表面含有约 $2.0 \mathrm{wt} \%$ 的 $\mathrm{Ce}$ 元素以及 $3.2 \mathrm{wt} \%$ 的 $\mathrm{Ti}$ 元 素(图 S5 和图 S6), 并且各种元素在薄膜表面分布均 匀(图 3)。

从 PEDOT 和 PEDOT:Ce $@ \mathrm{TiO}_{2}$ 的 $\mathrm{AFM}$ 二维和三 维高度图中能更直观地观察到两种薄膜表面的微观 形貌(图 S7)。通过 AFM 测得的 PEDOT:Ce@ $\mathrm{TiO}_{2}$ 的 表面粗粘度 $R a$ 为 $25.430 \mathrm{~nm}$, 明显高于 $\operatorname{PEDOT}(R a=$ $13.389 \mathrm{~nm})$, 说明 $\mathrm{Ce} @ \mathrm{TiO}_{2}$ 的复合对 PEDOT:Ce $@ \mathrm{TiO}_{2}$ 的表面形貌有显著影响。相比于 PEDOT 薄膜的 AFM 高度图和相图(图 2(b,c)), PEDOT:Ce@ $\mathrm{TiO}_{2}$ 的高度图 中(图 2(e)) 可明显观察到明暗区分, 其中条状亮白 部分是由薄膜中纳米棒状结构产生的高度差引起的, 而在 PEDOT:Ce@ $\mathrm{TiO}_{2}$ 的 $\mathrm{AFM}$ 相图中(图 2(f))未能 观察到因高度差而引起的明暗区分, 据此可以推测 在该薄膜材料中不存在明显的相分离现象。

如图 4(a)所示, 水滴在 PEDOT 薄膜的接触角为 $53.9^{\circ}$, 说明 PEDOT 薄膜具有亲水的表面; 然而 PEDOT: $\mathrm{Ce} @ \mathrm{TiO}_{2}$ 的水滴接触角为 $140.6^{\circ}$, 具有很强 的疏水性(图 4(c))。如图 4(b)所示, PEDOT:Ce@ $@ \mathrm{TiO}_{2}$

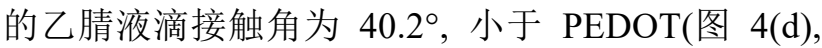
$55.2^{\circ}$ ), 表明 PEDOT:Ce@ $\mathrm{TiO}_{2}$ 对乙腈溶液的润湿性 优于 PEDOT 薄膜。

PEDOT:Ce@ $\mathrm{TiO}_{2}$ 很强的疏水性和对乙腈溶液 较好的润湿性可归因于其特殊的表面微/纳结构, 这 一性质暗示 PEDOT: $\mathrm{Ce} @ \mathrm{TiO}_{2}$ 在乙腈溶液等
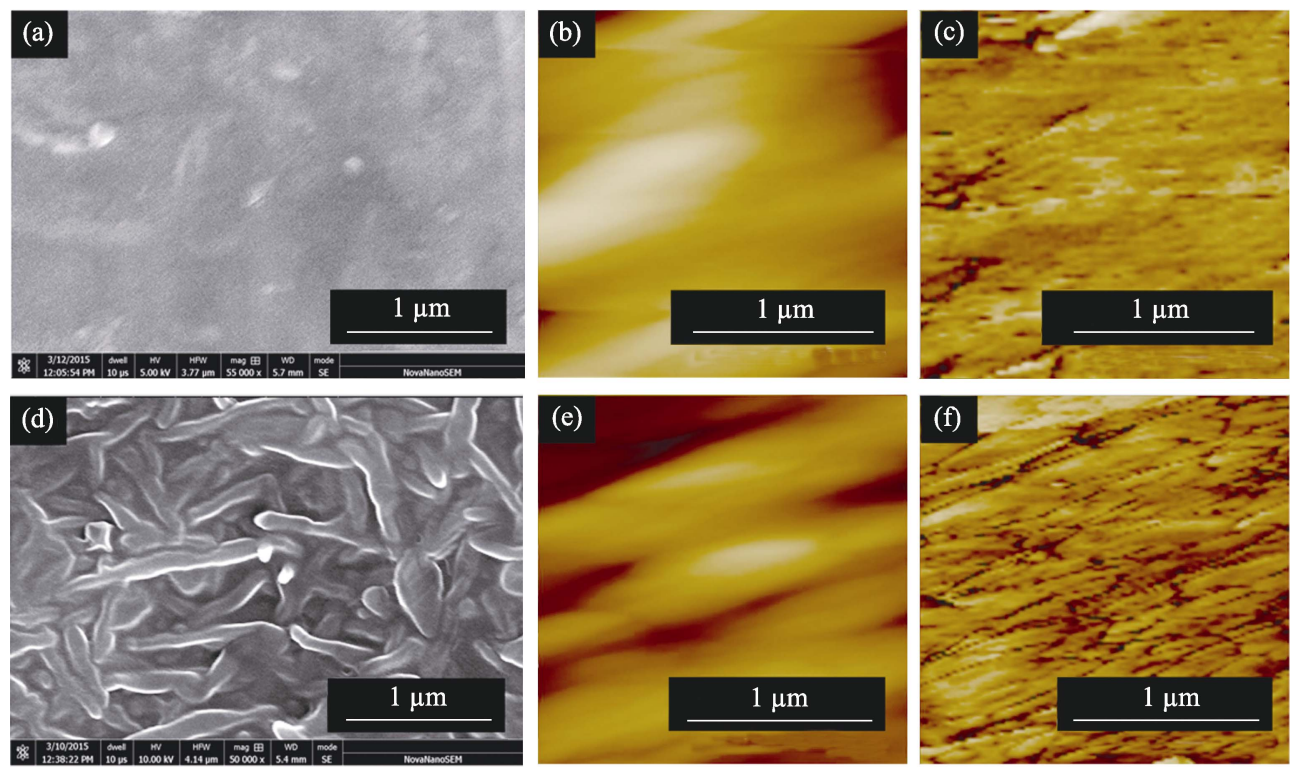

图 2 PEDOT 薄膜的(a)SEM 照片、(b)AFM 二维高度图、(c)AFM 二维相图和 PEDOT:Ce@ $\mathrm{TiO}_{2}$ 薄膜的 (d)SEM 照片、(e)AFM 二维高度图、(f)AFM 二维相图

Fig. 2 (a) SEM image, (b) AFM height sensor image (2D), (c) AFM tapping phase image (2D) of PEDOT film, and (d) SEM image, (e) AFM height sensor image (2D), (f) AFM tapping phase image (2D) of PEDOT:Ce@ $\mathrm{TiO}_{2}$ film 

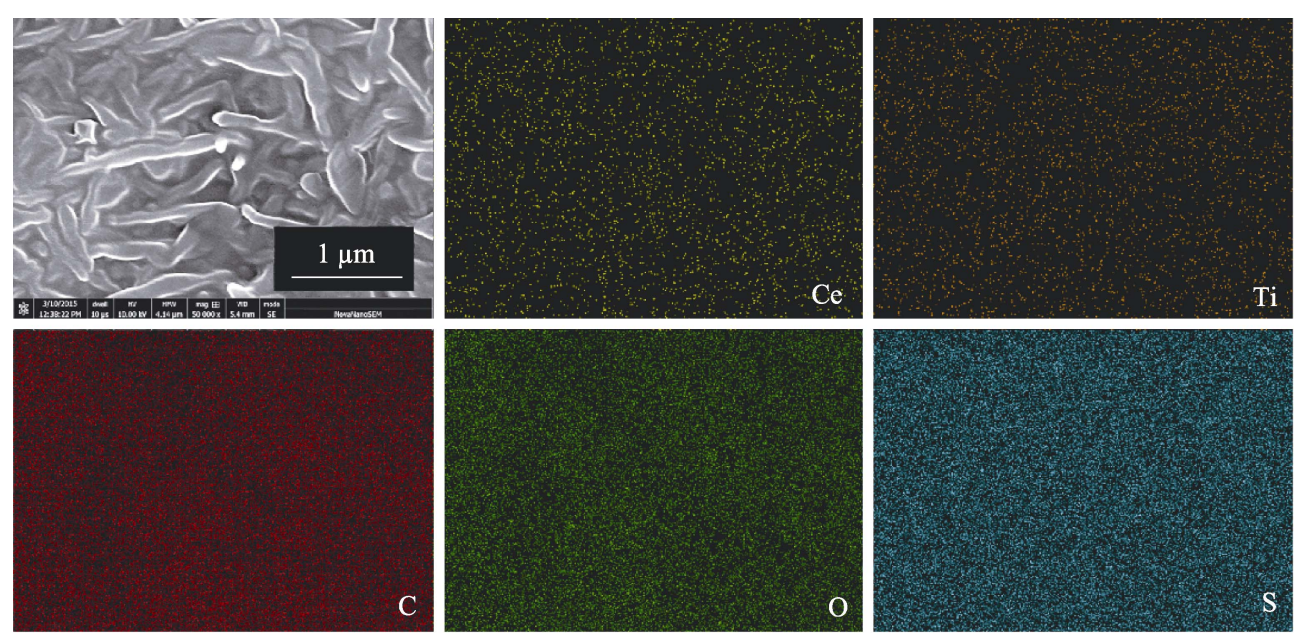

图 3 PEDOT:Ce@ $\mathrm{TiO}_{2}$ 薄膜的 SEM-mapping 照片

Fig. 3 SEM-mapping images of PEDOT:Ce@ $\mathrm{TiO}_{2}$ film

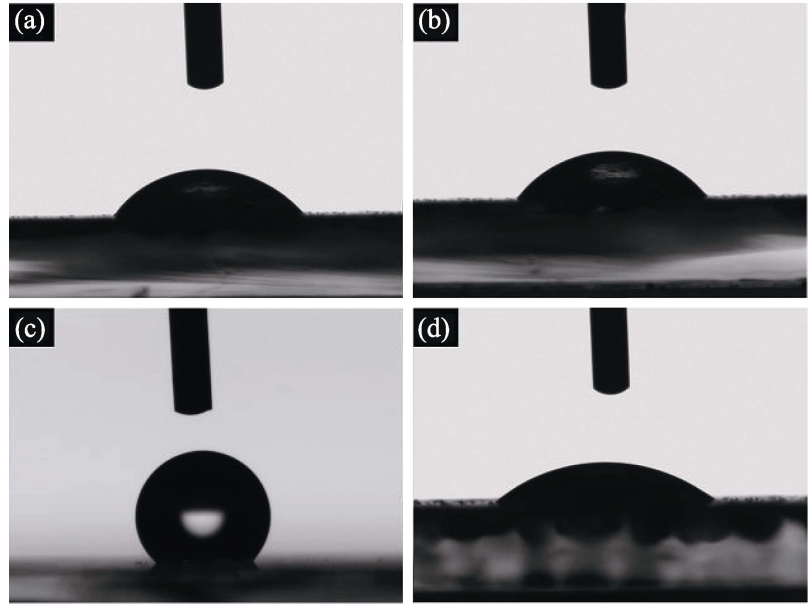

图 4 PEDOT 薄膜(a)和 PEDOT:Ce@ $\mathrm{TiO}_{2}$ 薄膜(c)的水滴接 触角照片; PEDOT 薄膜(b)和 PEDOT:Ce@ $\mathrm{TiO}_{2}$ 薄膜 $(\mathrm{d})$ 的乙 腈液滴接触角照片

Fig. 4 Images of water droplets on the surfaces of PEDOT film (a) and PEDOT:Ce@ $\mathrm{TiO}_{2}$ film (c), and images of ACN droplets on the surfaces of PEDOT film (b) and PEDOT: $\mathrm{Ce} @ \mathrm{TiO}_{2}$ film (d)

有机系电解液中可能有较好的电化学性能。我们发 现，通过在 PEDOT 薄膜中引入少量由 $\left[\mathrm{Ti}_{8} \mathrm{O}_{7}(\mathrm{HOEt})(\mathrm{OEt})_{21} \mathrm{Ce}\right]$ 转化的 $\mathrm{Ce} @ \mathrm{TiO}_{2}$ 就能显著 改变薄膜材料的润湿性能, 该方法未来还有可能应 用在制备超疏水涂层等新材料上。

\section{2 薄膜的电化学性能分析}

PEDOT 和 PEDOT:Ce $@ \mathrm{TiO}_{2}$ 电极的 $\mathrm{CV}$ 曲线如 图 S8 所示, 在扫描速率为 $100 \mathrm{mV} / \mathrm{s}$, 电压窗口为 $-0.8 \sim 1.5 \mathrm{~V}$ 时, PEDOT 电极有一对还原氧化峰(氧化 电位 $E p c=-0.52 \mathrm{~V}$, 还原电位 $E p a=0.27 \mathrm{~V}$ ), 而 PEDOT:Ce@ $\mathrm{TiO}_{2}$ 电极没有明显的氧化还原峰。如图 S9 所示, PEDOT 和 PEDOT:Ce@ $\mathrm{TiO}_{2}$ 都是阴极电致 变色材料 ${ }^{[34]}$, PEDOT 颜色从 $-0.8 \mathrm{~V}$ 时的深紫色变为
$0.8 \mathrm{~V}$ 时的浅蓝色, 而 PEDOT: $\mathrm{Ce} @ \mathrm{TiO}_{2}$ 从 $-0.8 \mathrm{~V}$ 的 蓝灰色变为 $0.8 \mathrm{~V}$ 的浅蓝色。在着色状态下, PEDOT:Ce@ $\mathrm{TiO}_{2}$ 的吸收峰 $(503 \mathrm{~nm})$ 相比于 PEDOT $(567 \mathrm{~nm})$ 发生了蓝移。

如图 5(a,b)所示, 在电压窗口为 $-0.2 \sim 0.8 \mathrm{~V}$, 扫描速 率为 $10 \mathrm{mV} / \mathrm{s}$ 时, PEDOT 和 PEDOT:Ce $@ \mathrm{TiO}_{2}$ 的 $\mathrm{CV}$ 曲 线均接近矩形, 表现出较好的电容性能, 随着扫描速 率的提高 $\mathrm{CV}$ 曲线逐渐扭曲为梭形。PEDOT:Ce@ $\mathrm{TiO}_{2}$ 的循环伏安曲线面积大于 PEDOT, 表明 PEDOT:Ce $@ \mathrm{TiO}_{2}$ 的储电能力比 PEDOT 更强 ${ }^{[35-39]}$ 。如 图 5(c,d)所示, PEDOT 和 PEDOT:Ce $@ \mathrm{TiO}_{2}$ 在电流密 度分别为 $1 \sim 5 \mathrm{~A} / \mathrm{g}$ 时的 $\mathrm{GCD}$ 曲线近似等腰三角形, 欧姆电压降很小。如图 S10(b)所示, 在电流密度为 $1 \mathrm{~A} / \mathrm{g}$ 时, PEDOT:Ce@ $\mathrm{TiO}_{2}$ 的质量比电容为 $71.2 \mathrm{~F} / \mathrm{g}$, 是相同条件下 PEDOT 的质量比电容 $(41.4 \mathrm{~F} / \mathrm{g})$ 的 1.7 倍; 当电流密度增加到 $5 \mathrm{~A} / \mathrm{g}$ 时, PEDOT:Ce@ $\mathrm{TiO}_{2}$ 的质量比电容保持在 $40.0 \mathrm{~F} / \mathrm{g}$, 是相同条件下 PEDOT 的质量比电容 $(25.0 \mathrm{~F} / \mathrm{g})$ 的 1.6 倍。如图 S10(a) 所示, PEDOT 具有更小的离子扩散阻抗, 但 PEDOT:Ce@ $\mathrm{TiO}_{2}$ 亲乙腈性以及特殊的纳米结构使 电解液更容易接触活性物质从而使得 PEDOT: $\mathrm{Ce} @ \mathrm{TiO}_{2}$ 电极材料展现出更好的电容性能; 如图 S11 所示, 经过 500 次恒流充放电循环, PEDOT: $\mathrm{Ce} @ \mathrm{TiO}_{2}$ 的电容衰减量为 $48.3 \%$, 较 PEDOT (电容衰减量为 $57.9 \%$ ) 具有更好的充放电循 环稳定性。

实验结果表明, PEDOT: $\mathrm{Ce} @ \mathrm{TiO}_{2}$ 不但是阴极电 致变色材料, 而且它作为超级电容器电极材料展现 出比 PEDOT 更好的电化学性能。因此我们采用 PEDOT: $\mathrm{Ce} @ \mathrm{TiO}_{2}$ 薄膜进一步组装了全固态电致变 色超级电容器。 


\section{3 全固态电致变色超级电容器的组装和性能}

聚(4,4',4"-三[4-(2-联噻吩基)苯基]胺) (PTBPTA) 是一种具有高光学对比度的阳极电致变色材料 ${ }^{[31]}$, 随着电压的变化, PTBPTA 能从还原态 $(0 \mathrm{~V})$ 的橙黄 色变为氧化态 $(1.2 \mathrm{~V})$ 的深蓝色。将电化学聚合制备 的 PTBPTA 薄膜(制备步骤详见补充材料 S9), 与 PEDOT:Ce@ $\mathrm{TiO}_{2}$ 薄膜匹配, 组装全固态电致变色 超级电容器原型器件。

如图 6(a) 所示, 该原型器件的一极以聚 (4,4',4"-三[4-(2-联噻吩基)苯基]胺)(PTBPTA)薄膜 电极为基底, 另一极以 PEDOT: $\mathrm{Ce} @ \mathrm{TiO}_{2}$ 薄膜电极
为基底，在两电极上进一步结合活性碳材料并以 高氯酸锂/聚甲基丙烯酸甲酯/聚碳酸酯/乙腈 $\left(\mathrm{LiClO}_{4} / \mathrm{PMMA} / \mathrm{PC} / \mathrm{ACN}\right)$ 作为全固态电解质 (制备 步骤详见补充材料 S10)。

如图 S13 所示, 该器件的 $\mathrm{CV}$ 曲线随着扫速的 提高逐步扭曲成梭形, 并且器件在不同电流密度下 的 GCD 曲线都有较明显的欧姆电压降，随着测试 电流密度的加大, 欧姆电压降也逐渐增大, 说明器 件的内部阻抗较高。如图 S14 所示, 当电流密度为 $0.21 \mu \mathrm{A} / \mathrm{cm}^{2}(1 \mathrm{~A} / \mathrm{g})$ 时, 器件的比容量为 $10.9 \mathrm{mF} / \mathrm{cm}^{2}(52.0 \mathrm{~F} / \mathrm{g})$; 当电流密度为 $1.05 \mu \mathrm{A} / \mathrm{cm}^{2}$
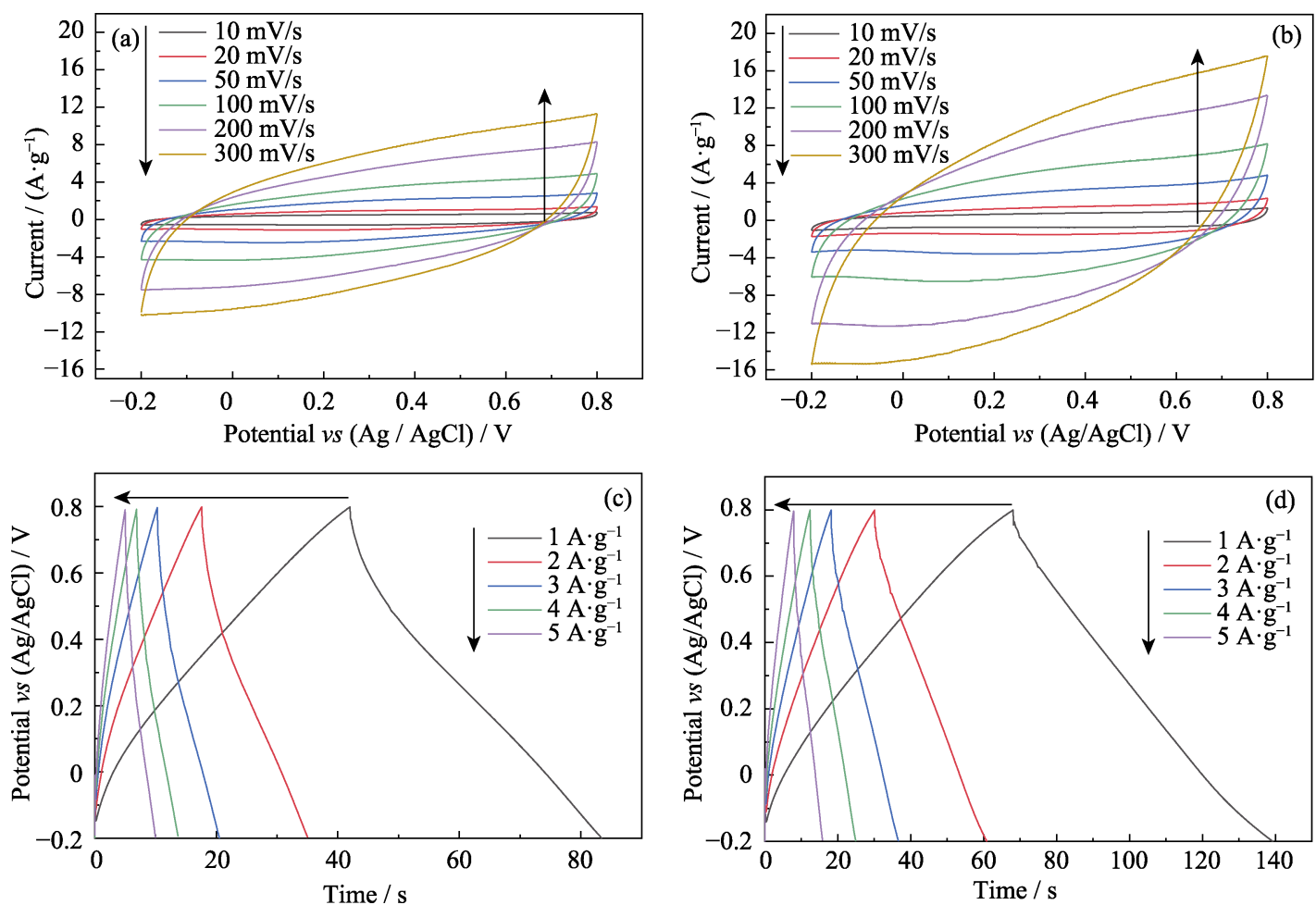

图 5 (a) PEDOT 和(b) PEDOT:Ce@ $\mathrm{TiO}_{2}$ 的循环伏安曲线; (c) PEDOT 和(d) PEDOT:Ce@ $\mathrm{TiO}_{2}$ 的恒流充放电曲线 Fig. 5 CV curves of (a) PEDOT and (b) PEDOT:Ce@ $\mathrm{TiO}_{2}$; Galvanostatic charge/discharge curves of (c) PEDOT and (d) PEDOT:Ce@ $\mathrm{TiO}_{2}$
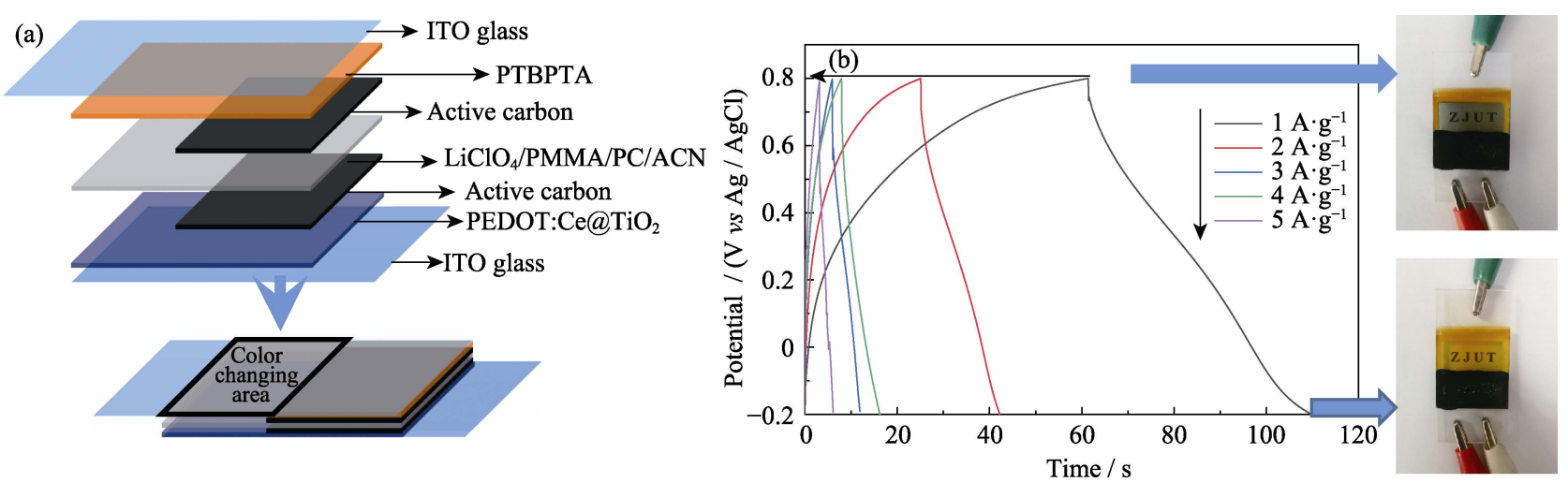

图 6 (a)电致变色超级电容器的结构示意图, (b) 恒流充放电曲线以及充电完成时和放电完成时的照片

Fig. 6 (a) Schematic, (b) galvanostatic charge/discharge curves of electrochromic supercapacitor, and optical photos under the completing of charging and discharging. 
$(5 \mathrm{~A} / \mathrm{g})$ 时, 器件的比容量为 $6.22 \mathrm{mF} / \mathrm{cm}^{2}(29.6 \mathrm{~F} / \mathrm{g})$ 。 如图 6(b) 所示, 当器件正负极两端电压为 $0.8 \mathrm{~V}$ 时完 成充电, 器件的变色区域呈现为墨绿色; 当正负极 两端电压为 $-0.2 \mathrm{~V}$ 时完成放电, 器件的变色区域呈 现为亮黄色。

\section{3 结论}

将铈钛氧簇 $\left[\mathrm{Ti}_{8} \mathrm{O}_{7}(\mathrm{HOEt})(\mathrm{OEt})_{21} \mathrm{Ce}\right]$ 和 PEDOT 低聚物的混合溶液通过滴涂-二次聚合成膜制得了 有特殊纳米沟壑表面的复合薄膜材料 PEDOT:Ce@ $\mathrm{TiO}_{2}$, 该材料具有很强的疏水性和对 乙腈溶液较好的润湿性。实验证明, PEDOT:Ce@ $\mathrm{TiO}_{2}$ 薄膜既能被用作阴极电致变色材料又可以作为超级 电容器电极材料, 展现出较 PEDOT 薄膜更优良的 电化学性能。我们采用 PEDOT:Ce@ $\mathrm{TiO}_{2}$ 薄膜进一 步组装了全固态电致变色超级电容器原型器件, 充 电完成时器件的变色区域呈现墨绿色, 放电完成时 器件的变色区域呈现亮黄色。

\section{补充材料:}

本文相关补充材料可登陆 https://doi.org/10. 15541/jim20190157 查看。

\section{参考文献:}

[1] SANTINA L M, ACHARYA S, D'ARCY J M. Low-temperature vapour phase polymerized polypyrrole nanobrushes for supercapacitors. J. Mater. Chem. A, 2017, 5: 11772-11780.

[2] YU C, WANG Y, CUI J, et al. Recent advances in the multimodification of $\mathrm{TiO}_{2}$ nanotube arrays and their application in supercapacitors. Acta Phys. -Chim. Sin., 2017, 33(10): 1944-1959.

[3] HUANG Y, LIANG J, CHEN Y. An overview of the applications of graphene-based materials in supercapacitors. Small, 2012, 8(12): 1805-1834.

[4] WANG K, WU H, MENG Y, et al. Conducting polymer nanowire arrays for high performance supercapacitors. Small, 2014, 10(1): 14-31.

[5] HOLZE R, WU Y P. Intrinsically conducting polymers in electrochemical energy technology: trends and progress. Electrochim. Acta, 2014, 122(9): 93-107.

[6] LIU H, ZHOU W, MA X, et al. Capacitive performance of electrodeposited PEDOS and a comparative study with PEDOT. Electrochim. Acta, 2016, 220: 340-346.

[7] ZHOU H, ZHI X, ZHAI H. High performance flexible supercapacitor based on electropolymerized poly(3,4-ethylenedioxythiophene) grown on superficial expansion-treated graphite. Org. Electron., 2018, 63: 149-158.

[8] HUANG Y, ZHU M, HUANG Y, et al. Multifunctional energy storage and conversion devices. Adv. Mater., 2016, 28(38): 8344-8364.

[9] KLANKOWSKI S A, PANDEY G P, MALEK G T, et al. Higher-power supercapacitor electrodes based on mesoporous ma- nganese oxide coating on vertically aligned carbon nanofibers. Nanoscale, 2015, 7(18): 8485-8494.

[10] YUKSEL R, CEVHER S C, CIRPAN A, et al. All-organic electrochromic supercapacitor electrodes. J. Electrochem. Soc., 2015, 162(14): A2805-A2810.

[11] SNOOK G A, KAO P, BEST A S. Conducting-polymer-based supercapacitor devices and electrodes. J. Power Sources, 2011, 196(1): 1-12.

[12] CHEN Y, ZHU X, YANG D, et al. A novel design of poly (3,4-ethylenedioxythiophene):poly (styrenesulfonate)/molybdenum disulfide/poly (3,4-ethylenedioxythiophene) nanocomposites for fabric microsupercapacitors with favourable performances. Electrochim. Acta, 2019, 298: 297-304.

[13] MA L, NIU H, CAI J, et al. Optical, electrochemical, photoelectrochemical and electrochromic properties of polyamide/graphene oxide with various feed ratios of polyamide to graphite oxide. $J$. Mater. Chem. C, 2014, 2(12): 2272-2282.

[14] YANG H, YU J, SEO H J, et al. Improved electrochromic properties of nanoporous $\mathrm{NiO}$ film by $\mathrm{NiO}$ flake with thickness controlled by aluminum. Appl. Surf. Sci., 2018, 461: 88-92.

[15] YUKSEL R, COSKUN S, GUNBAS G, et al. Nanocomposite electrochromic supercapacitor electrodes. J. Electrochem. Soc., 2017, 164(4): A721-A727.

[16] LÜ P, WANG Y, JI C, et al. Superelastic graphene aerogel/poly (3,4-ethylenedioxythiophene) $/ \mathrm{MnO}_{2}$ composite as compressiontolerant electrode for electrochemical capacitors. Materials, 2017, 10(12): 1353-1366.

[17] HOU Y, CHENG Y, HOBSON T, et al. Design and synthesis of hierarchical $\mathrm{MnO}_{2}$ nanospheres/carbon nanotubes/conducting polymer ternary composite for high performance electrochemical electrodes. Nano Lett., 2010, 10(7): 2727-2733.

[18] HERNANDEZ L A, RIVEROS G, MARTIN F, et al. Enhanced morphology, crystallinity and conductivity of poly(3,4-ethyldioxythiophene)/ ErGO composite films by in situ reduction of TrGO partially reduced on PEDOT modified electrode. Electrochim. Acta, 2017, 240: $155-162$.

[19] HAN Y, DING B, TONG H, et al. Capacitance properties of graphite oxide/poly(3,4-ethylene dioxythiophene) composites. Appl. Polym. Sci., 2011, 121(2): 892-898.

[20] ABDAH M A A M, RAHMAN N A, SULAIMA Y. Enhancement of electrochemical performance based on symmetrical poly (3,4-ethylenedioxythiophene) coated polyvinyl alcohol/graphene oxide/manganese oxide microfiber for supercapacitor. Electrochim. Acta, 2018, 259: 466-473.

[21] WU X, WANG Q, ZHANG W, et al. Preparation of all-solid-state supercapacitor integrated with energy level indicating functionality. Synthetic Met., 2016, 220: 494-501.

[22] CHEN Y, WANG Y, SUN P, et al. Nickel oxide nanoflake-based bifunctional glass electrodes with superior cyclic stability for energy storage and electrochromic applications. J. Mater. Chem. A, 2015, 3: 20614-20618.

[23] CHAVAN H S, HOU BO, AHMED A T A, et al. Nanoflake Ni$\mathrm{MoO}_{4}$ based smart supercapacitor for intelligent power balance monitoring. Energ. Mat. Sol. C, 2018, 185: 166-173.

[24] CARBAS B B, TEKIN B. Poly(3,4-ethylenedioxythiophene) electrode grown in the presence of ionic liquid and its symmetrical electrochemical supercapacitor application. Polym. Bull., 2018, 75: 1547-1652.

[25] LE T, BIDAN G, GENTILE P, et al. Understanding the energy storage mechanisms of poly(3,4-ethylenedioxythiophene)-coated silicon nanowires by electrochemical quartz crystal microbalance. Materials Letter, 2019, 240: 59-61. 
[26] NARESH V, ELIAS L, MARTHA S K. Poly (3,4-ethylenedioxythiophene) coated lead negative plates for hybrid energy storage systems. Electrochim. Acta, 2019, 301: 183- 191.

[27] HARRIS A, MINETT A, AITCHISON P, et al. Compositional effects of PEDOT-PSS/single walled carbon nanotube films on supercapacitor device performance. J. Mater. Chem., 2011, 21(40): 15987-15994.

[28] LIU H, LIU X, MU S, et al. A novel fabrication approach for three-dimensional hierarchical porous metal oxide/carbon nanocomposites for enhanced solar photocatalytic performance. Catal. Sci. Technol., 2017, 7: 1965-1970.

[29] KIM H, KIM K, CHOI D, et al. Evaluation of a reliable electrochromic device based on PEDOT:PSS- $\mathrm{TiO}_{2}$ heterostructure fabricated at low temperature. Ionics, 2017, 23: 2465-2474.

[30] MOLINA P, KOZMA K, SANTALA M, et al. Aqueous bismuth titanium-oxo sulfate cluster speciation and crystallization. Angew. Chem. Int. Ed., 2017, 56: 16277-16281.

[31] LÜ Y, DU W, REN Y, et al. An integrated electrochromic supercapacitor based on nanostructured Er-containing titania using an Er(III)-doped polyoxotitanate cage. Inorg. Chem. Front., 2016, 3: 1119-1123.

[32] LÜ Y, YAO M, HOLGADO J P, et al. A low-temperature singlesource route to an efficient broad-band cerium (III) photocatalyst using a bimetallic polyoxotitanium cage. $R S C A d v$., 2013, 3(33): 13659-13662.
[33] ZHOU H, YAO W, LI G, et al. Graphene/poly(3,4-ethylenedioxythiophene) hydrogel with excellent mechanical performance and high conductivity. Carbon, 2013, 59: 495-502.

[34] LIU G, YANG X, BONNEFONT A, et al. Conjugated hybrid films based on a new polyoxotitanate monomer. Chem. Commun., 2018, 54: 14132-14135.

[35] DU W, LÜ Y, CAI Z, et al. Flexible all-solid-state supercapacitor based on three-dimensional porous graphene/titanium-containing copolymer composite film. Acta Phys. -Chim. Sin., 2017, 33(9): 1828-1837.

[36] DU W, LÜ Y, LU H, et al. Surface modification by graphene oxide: an efficient strategy to improve the performance of activated carbon based supercapacitors. Chinese Chem. Lett., 2017, 28(12): 2285-2289.

[37] SONG Z, DUAN H, LI L. High-energy flexible solid-state supercapacitors based on $\mathrm{O}, \mathrm{N}$, S-tridoped carbon electrodes and a $3.5 \mathrm{~V}$ gel-type electrolyte. Chem. Eng. J., 2019, 372: 1216-1225.

[38] YAN J, ZHU D, LÜ Y, WEI X, et al. Water-in-salt electrolyte ion-matched N/O codoped porous carbons for high-performance supercapacitors. Chinese Chem. Lett., 2019, DOI: 10.1016/j.cclet. 2019.05.035.

[39] SONG Z, DUAN H, ZHU D, et al. Ternary-doped carbon electrodes for advanced aqueous solid-state supercapacitors based on a "water-in-salt" gel electrolyte J. Mater. Chem. A, 2019, DOI: 10. 1039/C9TA02690H. 
补充材料:

\title{
新型多功能无机/有机复合薄膜的制备及电化学性能研究
}

\author{
陈 钧 ${ }^{1}$, 马培华 ${ }^{2}$, 张 诚 ${ }^{1}$, 劳伦 . 鲁尔曼 ${ }^{3}$, 吕耀康 ${ }^{1}$
}

(1. 浙江工业大学 化学工程学院, 杭州 310014; 2. 绍兴金冶环保科技有限公司, 绍兴 312073; 3. 斯特拉斯堡大学 化学研究所, 斯特拉斯堡 67081, 法国)

\section{S1 实验原料与试剂}

3,4 -乙烯二氧噻吩(EDOT, 无水氯化铈, 钛酸四 乙酯，购自萨恩化学技术(上海)有限公司, $98 \%$ ); 对 甲苯磺酸铁(购自阿拉丁试剂(上海)有限公司, 98\%); 高氯酸锂 $\left(\mathrm{LiClO}_{4}\right.$, 购自阿拉丁试剂(上海)有限公司, 99.9\%); 聚甲基丙烯酸甲酯(PMMA)，分子量 90 万， 购自东莞双富塑胶有限公司; 乙腈 $(\mathrm{ACN})$, 二氯甲 烷(DCM), 碳酸丙烯酯(PC), 四氢呋喃(THF)无水乙 醇, 购自上海凌峰化学试剂有限公司, 均为分析纯 且使用前未经任何处理。

\section{S2 PEDOT 低聚物溶液的制备}

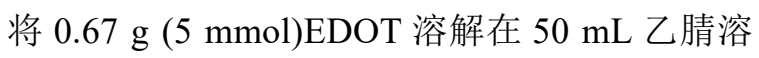
剂中并置于圆底烧瓶内, 以对甲苯磺酸铁 $(1.14 \mathrm{~g}$, $2 \mathrm{mmol}$ )为氧化剂, 溶于 $50 \mathrm{~mL}$ 乙腈溶剂中, 在 $20 \mathrm{~min}$ 内缓慢滴入盛有 EDOT 乙腈溶剂的圆底烧瓶 中, EDOT 发生了氧化聚合, 溶液逐渐变为深蓝色, 室温下反应 $2 \mathrm{~h}$ 后制得 PEDOT 低聚物溶液。

图 S1 为使用凝胶渗透色谱仪 (GPC) 测定的 PEDOT 低聚物溶液的相对分子质量分布曲线, 当 流出时间为 $22.0 \mathrm{~min}$, 在 $\lg M_{\mathrm{w}}=3.1 \sim 3.2$ 之间时, 其 积分分布曲线切线斜率最大, 即相对分子质量在此 区间的分布最多, PEDOT 的重均相对分子质量 $M_{\mathrm{w}}$ 为 $2833 \mathrm{~g} / \mathrm{mol}$, 相对分子质量分布 $M_{\mathrm{w}} / M_{\mathrm{n}}$ 为 1.44 ; 流出时间时为 $24.5 \mathrm{~min}$ 时在 $\lg M_{\mathrm{w}}=2.6 \sim 3.0$ 内, 相对 分子质量在此区间的分布最多, PEDOT 的重均相对 分子质量 $M_{\mathrm{w}}$ 为 $708 \mathrm{~g} / \mathrm{mol}$, 相对分子质量分布 $M_{\mathrm{w}} / M_{\mathrm{n}}$ 为 1.07 ; 流出时间时为 $25.9 \mathrm{~min}$, 在 $\lg M_{\mathrm{w}}=$ 2.5 2.6 之间时, 其积分分布曲线切线斜率最大, 即 相对分子质量在此区间的分布最多, PEDOT 的重均 相对分子质量 $M_{\mathrm{w}}$ 为 $310 \mathrm{~g} / \mathrm{mol}$, 相对分子质量分布 $M_{\mathrm{w}} / M_{\mathrm{n}}$ 为 $1.10 ;$ 在 $22.0 、 24.5 、 25.9 \mathrm{~min}$ 这三个流出 时间, PEDOT 相对分子质量分布较窄, 均匀性较 好。测试结果表明, 在所制备的 PEDOT 低聚物溶 液中, PEDOT 的聚合度主要分布在 3 10。

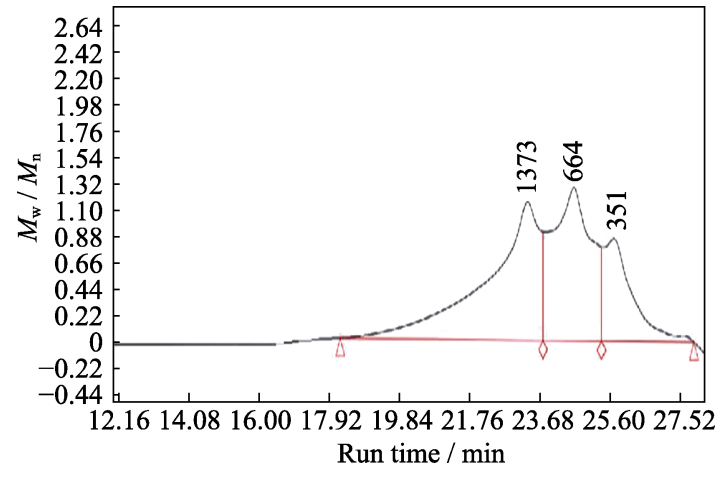

图 S1 流出时间为 12.0 28.0 min 内相对分子质量分布 Fig. S1 Relative molecular mass distribution with run time from 12.0 to $28 \mathrm{~min}$

\section{S3 PEDOT 薄膜的制备}

将制备好的 PEDOT 低聚物溶液（图 S2(a)）通 过滴涂法均匀覆盖于 ITO 表面, 置于 $60{ }^{\circ} \mathrm{C}$ 烘箱, $2 \mathrm{~h}$ 后用去离子水洗涤, 在 $60{ }^{\circ} \mathrm{C}$ 下再次烘干后制得 PEDOT 薄膜, 图 S2(d) 是该薄膜的照片。

\section{S4 铈钛氧簇 $\left[\mathrm{Ti}_{8} \mathrm{O}_{7}(\mathrm{HOEt})(\mathrm{OEt})_{21} \mathrm{Ce}\right]$ 的二氯甲烷溶液的制备}

将钛酸四乙酯 $(3.5 \mathrm{~mL}, 15.4 \mathrm{mmol})$, 无水氯化 铈 $(0.246 \mathrm{~g}, 1 \mathrm{mmol})$, 无水乙醇 $(7 \mathrm{~mL})$ 加入聚四氟乙 烯高压反应釜内,并在 $150{ }^{\circ} \mathrm{C}$ 反应 $48 \mathrm{~h}$, 冷却至室温 后获得淡黄色溶液, 溶液静置 $24 \mathrm{~h}$ 后获得橙黄色铈 钛氧簇 $\left[\mathrm{Ti}_{8} \mathrm{O}_{7}(\mathrm{HOEt})(\mathrm{OEt})_{21} \mathrm{Ce}\right]$ 晶体。将 $0.5 \mathrm{~g}$ $\left[\mathrm{Ti}_{8} \mathrm{O}_{7}(\mathrm{HOEt})(\mathrm{OEt})_{21} \mathrm{Ce}\right]$ 晶体溶解在 $10 \mathrm{~mL}$ 二氯甲烷 中配制 $0.03 \mathrm{~mol} / \mathrm{L}\left[\mathrm{Ti}_{8} \mathrm{O}_{7}(\mathrm{HOEt})(\mathrm{OEt})_{21} \mathrm{Ce}\right]$ 的二氯甲 烷溶液(图 S2(b))。

\section{S5 PEDOT:Ce@ $\mathrm{TiO}_{2}$ 薄膜的制备}

将 P E D O T 低聚物溶液和铈钛氧簇 $\left[\mathrm{Ti}_{8} \mathrm{O}_{7}(\mathrm{HOEt})(\mathrm{OEt})_{21} \mathrm{Ce}\right]$ 的二氯甲烷(DCM)溶液按体 积比 1:1 均匀混合, 将两者充分混合, 超声 $20 \mathrm{~min}$ 后通过滴涂的方法均匀覆盖于氧化铟锡(ITO)玻璃 

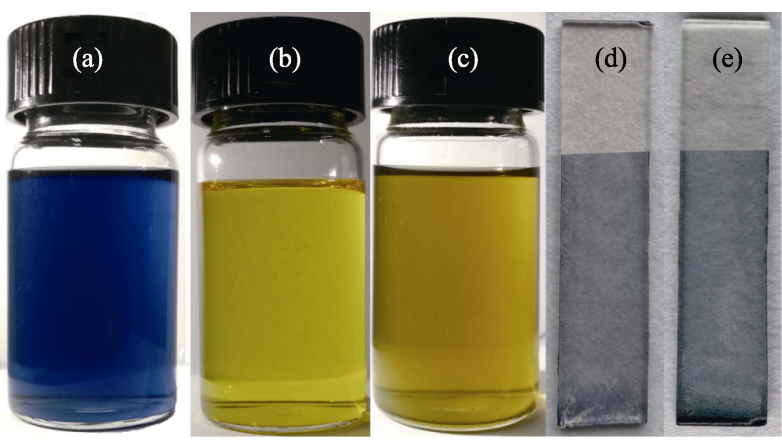

图 S2 (a) PEDOT 低聚物, (b) 铈钛氧簇 $\left[\mathrm{Ti}_{8} \mathrm{O}_{7}(\mathrm{HOEt})(\mathrm{OEt})_{21} \mathrm{Ce}\right]$, (c) 铈钛氧簇 $\left[\mathrm{Ti}_{8} \mathrm{O}_{7}(\mathrm{HOEt})(\mathrm{OEt})_{21} \mathrm{Ce}\right]$ 和 PEDOT 的 DCM 溶液, (d) PEDOT 薄膜(e) PEDOT: Ce@ $\mathrm{TiO}_{2}$ 薄 膜的照片

Fig. S2 Photographs of solution of PEDOT oligomer in DCM, (b) solution of polyoxotitanate cluster $\left[\mathrm{Ti}_{8} \mathrm{O}_{7}(\mathrm{HOEt})(\mathrm{OEt})_{21} \mathrm{Ce}\right]$ in DCM, (c) mixture solution of PEDOT and polyoxotitanate cluster $\left[\mathrm{Ti}_{8} \mathrm{O}_{7}(\mathrm{HOEt})(\mathrm{OEt})_{21} \mathrm{Ce}\right]$ in DCM, (d) PEDOT film (e) PEDOT: $\mathrm{Ce} @ \mathrm{TiO}_{2}$ film.

表面, 置于 $60{ }^{\circ} \mathrm{C}$ 烘箱, $2 \mathrm{~h}$ 后用去离子水洗涤, 在 $60{ }^{\circ} \mathrm{C}$ 下再次烘干后制得 PEDOT:Ce@ $\mathrm{TiO}_{2}$ 薄膜, 图 S2(e) 是 该薄膜的照片。

\section{S6 薄膜的红外光谱分析}

如图 S3 所示, PEDOT 薄膜在 $1640 \mathrm{~cm}^{-1}$ 处的吸 收峰为噻吩环上 $\mathrm{C}=\mathrm{C}$ 的振动吸收峰; 在 1440 和 $1360 \mathrm{~cm}^{-1}$ 处的吸收峰分别归属于噻吩环上的 $\mathrm{C}=\mathrm{C}$ 和 $\mathrm{C}-\mathrm{C}$ 的不对称伸缩振动; 在 $1120 \mathrm{~cm}^{-1}$ 处为二氧 次乙基环的伸缩振动吸收峰, 其弯曲振动的吸收峰 在 1190 和 $1040 \mathrm{~cm}^{-1}$ 处; 1010 和 $812 \mathrm{~cm}^{-1}$ 处的振动吸 收峰属于噻吩环上的 $\mathrm{C}-\mathrm{S}-\mathrm{C}$ 键的弯曲振动。PEDOT: $\mathrm{Ce} @ \mathrm{TiO}_{2}$ 薄膜的红外光谱中由 PEDOT 薄膜中存在 的类似官能团组成, 但对应于噻吩环上的 $\mathrm{C}=\mathrm{C}$ 和 C $-\mathrm{C}$ 伸缩振动的峰值蓝移至 1490 和 $1370 \mathrm{~cm}^{-1}$, 对应 的 C-O-C 键峰值红移至 1060 和 $893 \mathrm{~cm}^{-1}$, 证明了 PEDOT:Ce@ $\mathrm{TiO}_{2}$ 复合薄膜的形成。

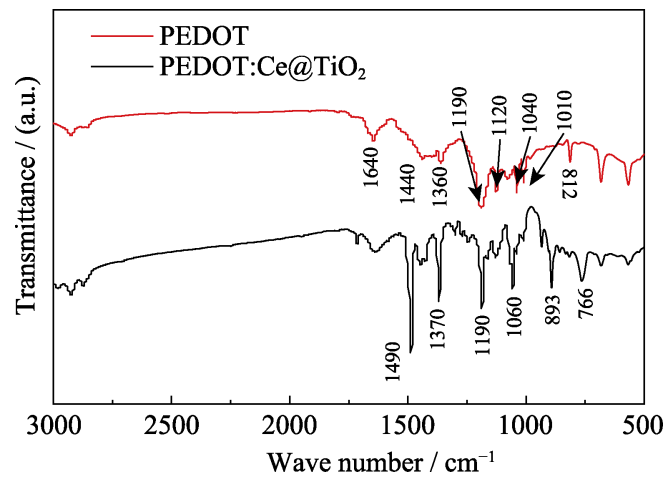

图 S3 PEDOT 和 PEDOT:Ce $@ \mathrm{TiO}_{2}$ 的 FT-IR 光谱图

Fig. S3 FT-IR spectra of PEDOT and PEDOT:Ce@ $\mathrm{TiO}_{2}$

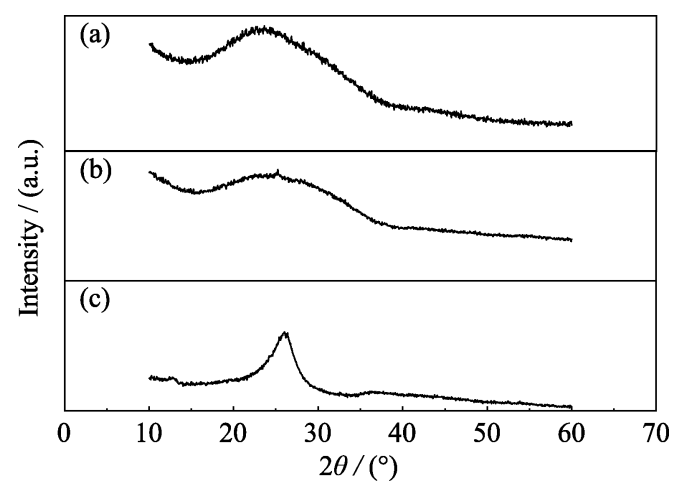

图 S4 (a) PEDOT:Ce@ $\mathrm{TiO}_{2}$ 粉末; (b) $\mathrm{Ce} @ \mathrm{TiO}_{2}$ 粉末和 (c) PEDOT 粉末的 XRD 图谱

Fig. S4 XRD patterns of (a) PEDOT:Ce@ $\mathrm{TiO}_{2}$ powder; (b) $\mathrm{Ce} @ \mathrm{TiO}_{2}$ powder and (c) PEDOT powder

\section{S7 X 射线能谱(EDS)分析}

分别对 PEDOT 和 PEDOT:Ce@ $\mathrm{TiO}_{2}$ 这两种薄膜 进行了 $X$ 射线能谱(EDS)分析。图 S5 为薄膜 PEDOT 的 EDS 能谱图, 图 S6 为薄膜 PEDOT:Ce@ $\mathrm{TiO}_{2}$ 的 EDS 能谱图。除了 PEDOT 所含的 $\mathrm{C} 、 \mathrm{O} 、 \mathrm{~S}$ 元素以 外, $\mathrm{Ce} 、 \mathrm{Ti}$ 的存在证明了 PEDOT:Ce@ $\mathrm{TiO}_{2}$ 复合薄膜 的成功制备, 其中各元素的质量百分含量如表 S1 所示。如图 3 所示, PEDOT:Ce $@ \mathrm{TiO}_{2}$ 薄膜中, Ce、

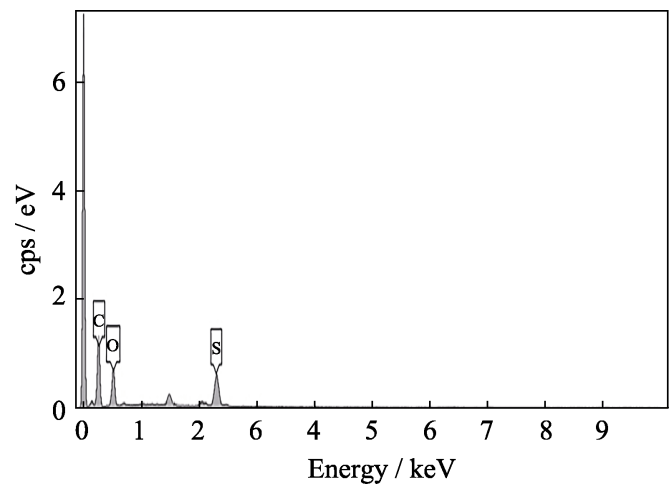

图 S5 PEDOT 薄膜的 EDS 能谱图

Fig. S5 EDS spectrum of film PEDOT

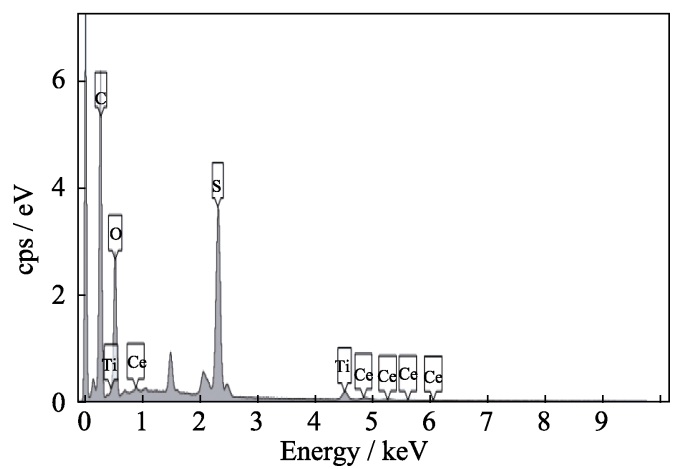

图 S6 PEDOT:Ce@ $\mathrm{TiO}_{2}$ 薄膜的 EDS 能谱图

Fig. S6 EDS spectrum of film PEDOT:Ce@ $\mathrm{TiO}_{2}$ 
表 S1 PEDOT 和 PEDOT:Ce@ $\mathrm{TiO}_{2}$ 薄膜中碳, 氧, 硫, 钛和铈原子的元素质量百分比

Table S1 Mass percentages of the carbon, oxygen, sulfur, titanium and cerium atoms in PEDOT and PEDOT: Ce@ $\mathrm{TiO}_{2}$ film

\begin{tabular}{cccccc}
\hline Film & $\mathrm{C} / \mathrm{wt} \%$ & $\mathrm{O} / \mathrm{wt} \%$ & $\mathrm{~S} / \mathrm{wt} \%$ & $\mathrm{Ti} / \mathrm{wt} \%$ & $\mathrm{Ce} / \mathrm{wt} \%$ \\
\hline PEDOT & 62.38 & 20.13 & 17.49 & - & - \\
PEDOT: Ce@ $\mathrm{TiO}_{2}$ & 60.01 & 18.26 & 15.91 & 3.18 & 2.01 \\
\hline
\end{tabular}
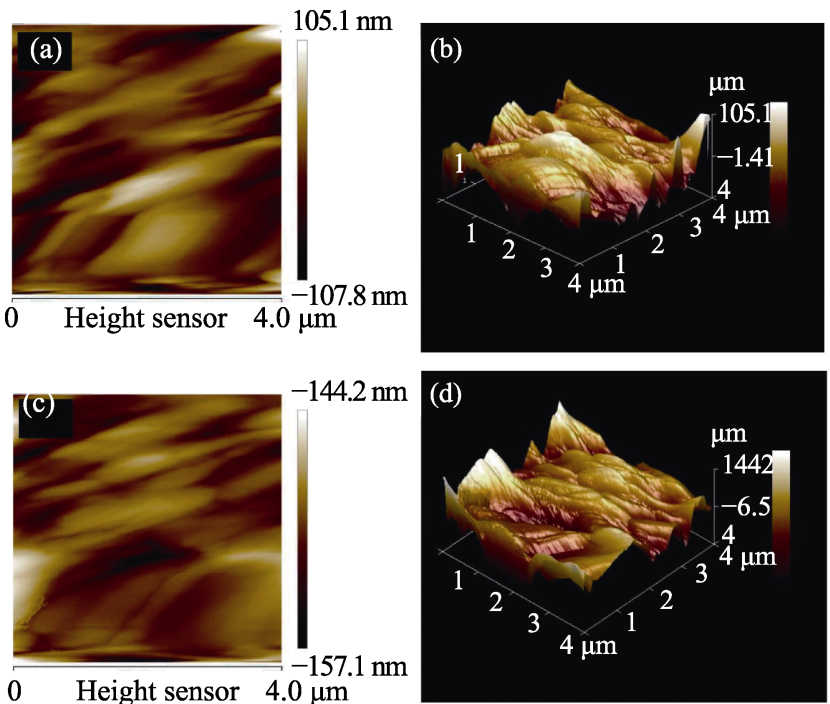

图 S7 (a) PEDOT 薄膜的 AFM 二维高度图, (b) AFM 三维高 度图; (c) PEDOT:Ce@ $\mathrm{TiO}_{2}$ 薄膜的 AFM 二维高度图, (d) AFM 三维高度图

Fig. S7 (a) AFM height sensor image (2D), (b) AFM height sensor image (3D) of PEDOT film; (c) AFM height sensor image (2D), (d) AFM height sensor image (3D) of PEDOT:Ce@ $\mathrm{TiO}_{2}$ film

Ti、C、O、S 等元素分布均匀。

\section{S8 薄膜材料的电化学性能测试}

循环伏安 $(\mathrm{CV})$ 测试采用三电极体系, 以 PEDOT 和 PEDOT:Ce@ $\mathrm{TiO}_{2}$ 薄膜电极为工作电极, 抛光过的铂丝为辅助电极, 双液接型 $\mathrm{Ag} / \mathrm{AgCl}$ 电极 (3 $\mathrm{mol} / \mathrm{L}$ 的氯化钾水溶液作为第一液接, 体系中的 电解液作为第二液接) 作为参比电极。溶剂为乙腈 $(\mathrm{ACN})$, 支持电解质为高氯酸锂 $(0.1 \mathrm{~mol} / \mathrm{L}$ 的 $\left.\mathrm{LiClO}_{4}\right)$ 。循环伏安 $(\mathrm{CV})$ 测试的电压窗口为 $-0.8 \sim 1.5 \mathrm{~V}$, 扫描速率为 $0.1 \mathrm{~V} / \mathrm{s}$, PEDOT 薄膜和 PEDOT:Ce@ $\mathrm{TiO}_{2}$ 薄膜的 CV 曲线如图 S8 所示。

如图 S8 所示, PEDOT 薄膜在 $0.21 \mathrm{~V}$ 左右出现 了一个氧化峰, 在 $-0.53 \mathrm{~V}$ 左右出现了一个明显的 还原峰, 对应于 PEDOT 氧化态和还原态之间的变 化; 然而 PEDOT:Ce@ $\mathrm{TiO}_{2}$ 薄膜的氧化还原峰并不

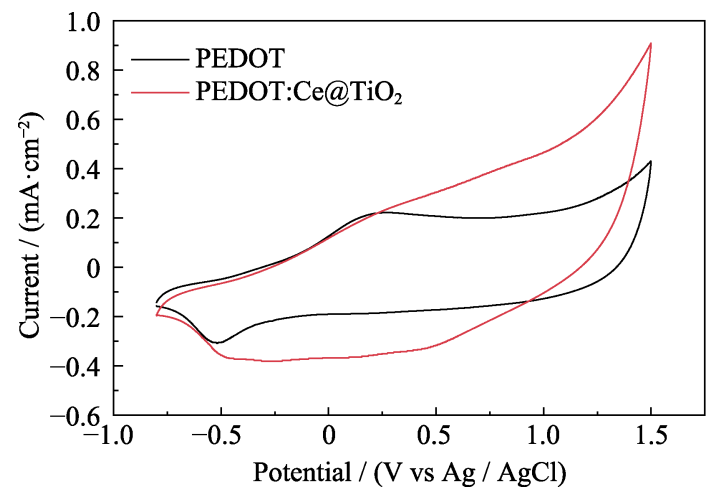

图 S8 在扫描速率 $100 \mathrm{mV} / \mathrm{s}$ 下 PEDOT 和 PEDOT:Ce@ $\mathrm{TiO}_{2}$ 薄膜在 $0.1 \mathrm{~mol} \cdot \mathrm{L}^{-1} \mathrm{LiClO}_{4} / \mathrm{ACN}$ 溶液中的循环伏安曲线 Fig. S8 Cyclic voltammetry curves of PEDOT and PEDOT:Ce@ $\mathrm{TiO}_{2}$ films in $0.1 \mathrm{~mol} / \mathrm{L} \mathrm{LiClO}_{4} / \mathrm{ACN}$ at scan rate of $100 \mathrm{mV} / \mathrm{s}$

明显, 这是由于在 PEDOT 与 $\mathrm{Ce} @ \mathrm{TiO}_{2}$ 复合后的循 环伏安过程中, 除了 PEDOT 发生了氧化还原反应 外, $\mathrm{Ce} @ \mathrm{TiO}_{2}$ 也发生了 $\mathrm{Li}^{+}$离子的嵌入脱出等反应, 多种氧化还原反应重叠在一起, 掩盖了 PEDOT 氧 化还原峰。

光谱电化学测试采用紫外-可见分光光度计和 电化学工作站联用技术, 实时测定薄膜的光谱电化 学性能, 以所制备的 PEDOT 和 PEDOT:Ce@ $\mathrm{TiO}_{2}$ 薄膜作为工作电极, 螺旋形铂丝作为对电极,
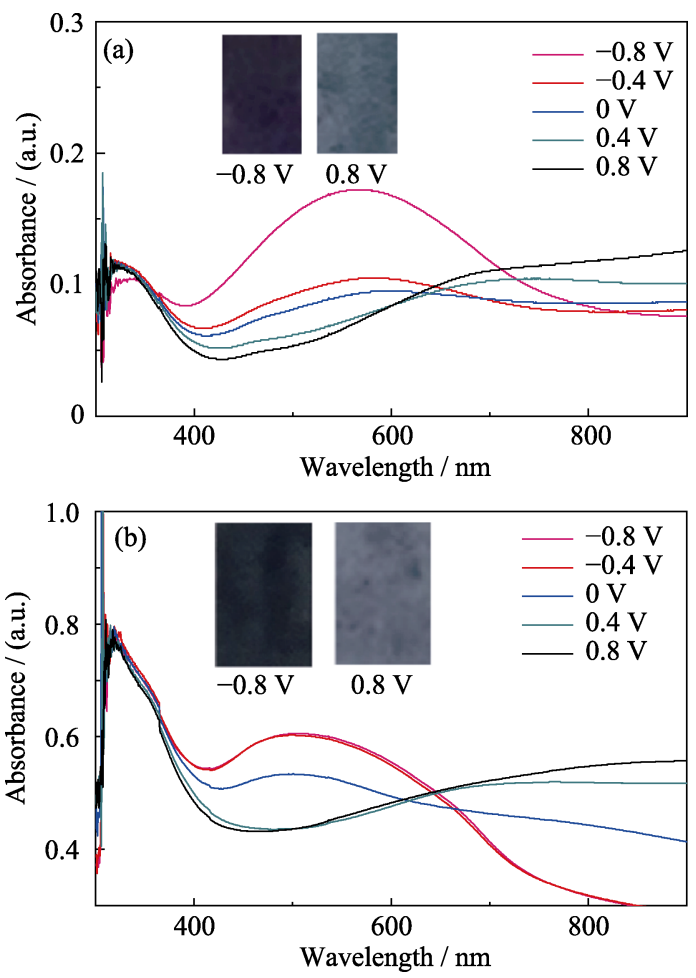

图 S9 (a) PEDOT 和(b) PEDOT:Ce@ $\mathrm{TiO}_{2}$ 薄膜在不同电压 下的紫外-可见光谱图和光学照片(插图)

Fig. S9 UV-Vis spectra and optical photos (inserts) of films under different potentialaption (a) PEDOT; (b) PEDOT:Ce@ $\mathrm{TiO}_{2}$ 
$\mathrm{Ag} / \mathrm{AgCl}$ 电极作为参比电极, $0.1 \mathrm{~mol} / \mathrm{L}$ 的 $\mathrm{LiClO}_{4} / \mathrm{ACN}$ 溶液作为电解液。

以规格为 $25 \mathrm{~mm} \times 30 \mathrm{~mm}$ 的 PEDOT 和 PEDOT: $\mathrm{Ce} @ \mathrm{TiO}_{2}$ 薄膜电极作为工作电极, 以规格 $30 \mathrm{~mm} \times$ $25 \mathrm{~mm}$ 的铂片作辅助电极, 以 $\mathrm{Ag} / \mathrm{AgCl}$ 电极为参比电 极, 以 $0.1 \mathrm{~mol} / \mathrm{L}$ 的 $\mathrm{LiClO}_{4} / \mathrm{ACN}$ 溶液作为电解液, 组 成三电极体系, 分别测试 PEDOT 和 PEDOT:Ce@ $\mathrm{TiO}_{2}$ 薄膜作为超级电容器电极材料的电化学性能。

$\mathrm{CV}$ 测试采用电压窗口为 $-0.2 \sim 0.8 \mathrm{~V}$, 扫描速率 分别为 $300 、 200 、 100 、 50 、 20 、 10 \mathrm{mV} / \mathrm{s}$; GCD 测 试采用与 $\mathrm{CV}$ 相同的电压窗口，电流密度分别为 1 、 2、3、4、5 A/g; EIS 测试在开路电位下进行。

电极材料的比容量均采用 GCD 测试进行计算 ${ }^{[2]}$, 其中三电极测试质量比容量计算公式为:

$$
C_{\mathrm{s}}=\frac{I \Delta t}{m \Delta V}
$$

其中, $C_{\mathrm{s}}$ 为材料的质量比容量 $(\mathrm{F} / \mathrm{g}) ; I$ 为横流充放电 电流 (A); $t$ 为放电时间 (s); $m$ 为电极活性物质质量 $(\mathrm{g}) ; V$ 为充放电去除欧姆电压降后的电压窗口 $(\mathrm{V})$ 。

如图 S10(a)所示, PEDOT 的 Nyquist 曲线与 PEDOT:Ce@ $\mathrm{TiO}_{2}$ 的 Nyquist 曲线相比拥有更加靠
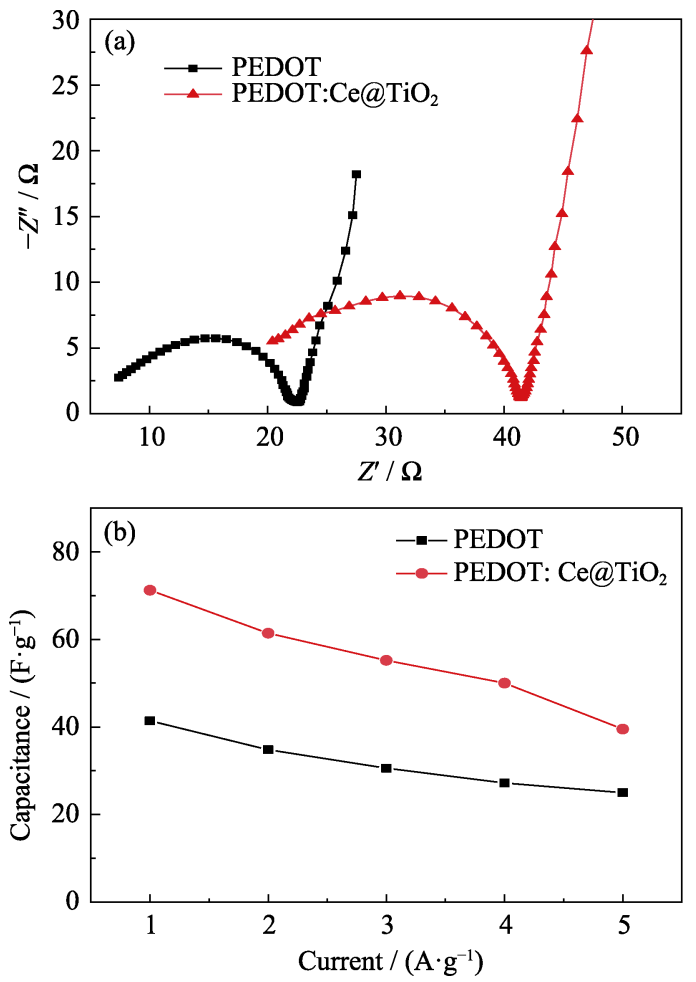

图 S10 (a) PEDOT 和 PEDOT:Ce@ $\mathrm{TiO}_{2}$ 的交流阻抗曲线; (b) 不同充放电电流密度下 PEDOT 和 PEDOT:Ce $@ \mathrm{TiO}_{2}$ 薄膜的 质量比容量

Fig. S10 (a) Nyquist plots of the impedance spectra of PEDOT and PEDOT: Ce@ $\mathrm{TiO}_{2}$; (b) Specific capacitance calculated from discharge curves of PEDOT and PEDOT: $\mathrm{Ce} @ \mathrm{TiO}_{2}$ films
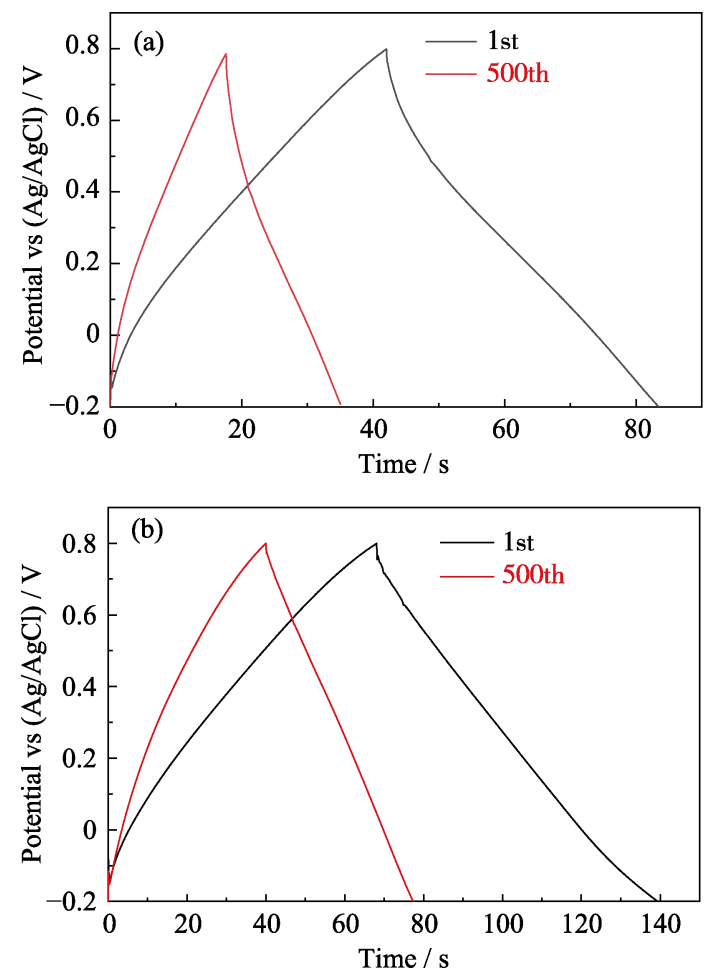

图 S11 (a) PEDOT 和(b) PEDOT:Ce@ $\mathrm{TiO}_{2}$ 薄膜在电流密度 为 $1 \mathrm{~A} / \mathrm{g}$ 时的充放电循环稳定性

Fig. S11 Cycling performance of PEDOT (a) and PEDOT:Ce@ $\mathrm{TiO}_{2}$ (b) film measured by charging and discharging it at $1 \mathrm{~A} / \mathrm{g}$

近 $Z$ "轴的低频部分曲线, 说明 PEDOT 具有较小的 离子扩散阻抗。交流阻抗高频区域的半圆大小与电 极表面的电荷转移阻抗正相关, PEDOT 材料的电荷 转移电阻略低于 PEDOT:Ce@ $\mathrm{TiO}_{2}$ 。这可能是由于 加入了导电性较差的 $\mathrm{Ce} @ \mathrm{TiO}_{2}$ 使得材料的电阻增 大, 影响了材料的离子传输与电子转移过程。

\section{S9 聚(4,4',4"-三[4-(2-联噻吩基)苯基] 胺) (PTBPTA)薄膜的制备}

如图 S12 所示, 采用电化学恒电位聚合法, 在 三电极体系中以 ITO 导电玻璃为工作电极(规格为 $25 \mathrm{~mm} \times 30 \mathrm{~mm}$ ), 铂片为辅助电极 (规格 $30 \mathrm{~mm} \times$ $25 \mathrm{~mm} \times 0.1 \mathrm{~mm}$ ), $\mathrm{Ag} / \mathrm{AgCl}$ 电极为参比电极 $(3 \mathrm{~mol} / \mathrm{L}$ 的氯化钾水溶液作为第一液接, 体系中的电解液作 为第二液接), 以 $0.75 \mathrm{mmol} / \mathrm{L}$ 的 4,4',4"-三[4-(2-联 噻吩基)苯基]胺(TBPTA) 为单体, 支持电解质为 $0.1 \mathrm{~mol} / \mathrm{L} \mathrm{TBAP}$ (四丁基高氯酸铵) 以二氯甲烷 $(D C M)$ 和乙腈 $(A C N)$ (体积比 DCM: $A C N$ 为 7:3) 作为 溶剂, 聚合电位为 $1.2 \mathrm{~V}$, 聚合电量为 $0.04 \mathrm{C}$ 。电聚 合反应完成后, 薄膜在相应空白溶液中负电位下脱 掺杂 $100 \mathrm{~s}$ ，再用乙腈淋洗，制得聚(4,4',4"-三[4-(2联噻吩基)苯基]胺) (PTBPTA)薄膜。 


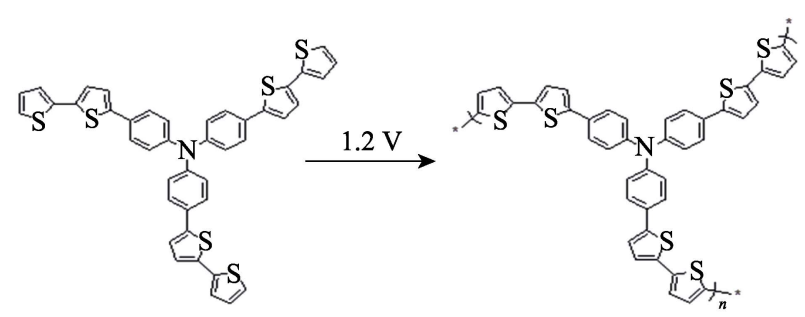

图 S12 PTBPTA 薄膜的电聚合路线

Fig. S12 Electro-polymerization route for PTBPTA

\section{S10 LiClO4/PMMA/PC/ACN 固体电 解质的制备方法和电致变色超级电容 器的组装}

将 $0.6 \mathrm{~g}$ 高氯酸锂 $\left(\mathrm{LiClO}_{4}\right), 1.4 \mathrm{~g}$ 聚甲基丙烯酸 甲酯(PMMA), $4 \mathrm{~g}$ 碳酸丙烯酯( $\mathrm{PC}$ )均匀混合, 置于 $80{ }^{\circ} \mathrm{C}$ 烘箱中溶胀反应 $24 \mathrm{~h}$, 呈凝胶状时加入 $14 \mathrm{~g}$ 乙腈溶剂( $\mathrm{ACN}$ ) 充分溶解, 置于 $80{ }^{\circ} \mathrm{C}$ 烘箱中继续 溶胀, 待乙腈溶剂挥发后形成透明无色胶状溶胶, 制得 $\mathrm{LiClO}_{4} / \mathrm{PMMA} / \mathrm{PC} / \mathrm{ACN}$ 固体电解质。

以活性炭为活性物质, 以乙炔黑为导电剂, 以 聚偏氟乙烯(PVDF)为粘结剂, 按质量比 $8: 1: 1$ 均 匀混合, 摚拌至粘稠状, 制得活性炭浆。分别在 PTBPTA 薄膜电极和 PEDOT:Ce@ $\mathrm{TiO}_{2}$ 薄膜电极表 面涂布活性炭浆并在 $60{ }^{\circ} \mathrm{C}$ 的烘箱中烘干, 由于电 极上的 PTBPTA 薄膜和 PEDOT:Ce@ $\mathrm{TiO}_{2}$ 薄膜都比 较薄, 导致其面积比电容不高, 涂布活性炭后能提 高 PEDOT:Ce@ $\mathrm{TiO}_{2}$ 薄膜电极和 PTBPTA 薄膜电极 的电容量。按如图 6(a)所示的方法将电极和固体电解 质组装在一起制成电致变色超级电容器原形器件。

\section{S11 电致变色超级电容器性能分析}

电致变色超级电容器的 $\mathrm{CV}$ 测试采用的电压窗 口为 $-0.2 \sim 0.8 \mathrm{~V}$ ，扫描速率分别为 300、200、100、 $50 、 20 、 10 \mathrm{mV} / \mathrm{s}$; GCD 测试采用与 $\mathrm{CV}$ 相同的电压 窗口，电流密度分别为 $1 、 2 、 3 、 4 、 5 \mathrm{~A} / \mathrm{g}$ 。

超级电容器的面积比容量采用 GCD 测试进行 计算 ${ }^{[2]}$, 计算公式为:

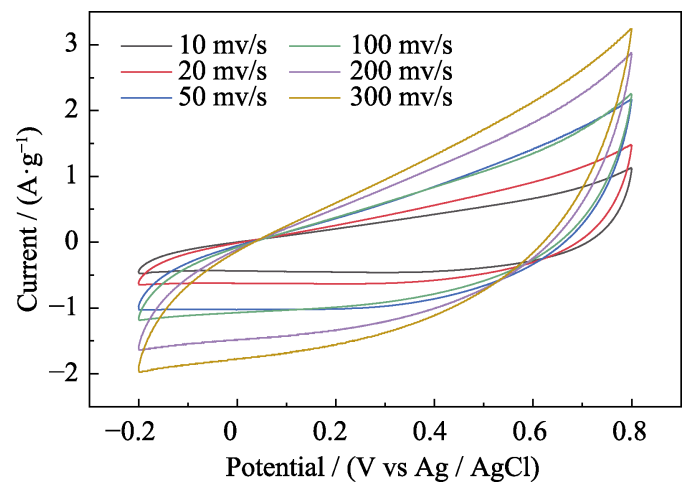

图 S13 电致变色超级电容器的循环伏安曲线

Fig. S13 CV curves of electrochromic supercapacitor

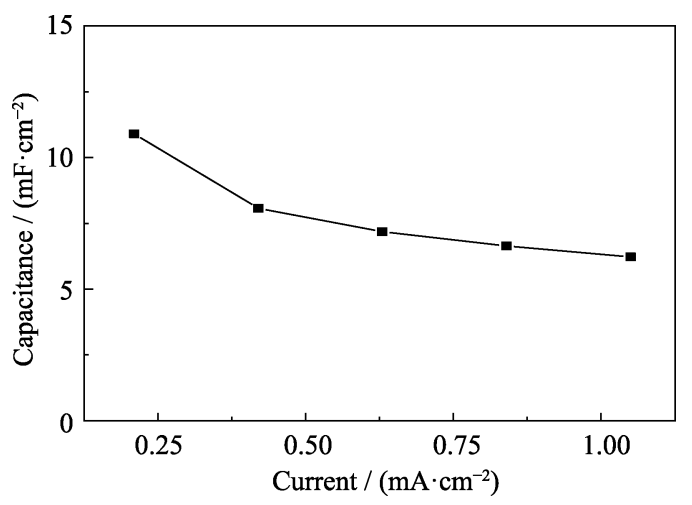

图 S14 不同电流密度下, 电致变色超级电容器的面积比 容量

Fig. S14 Specific capacitance calculated from discharge curves of electrochromic supercapacitor

$$
C_{\mathrm{s} 2}=\frac{I \Delta t}{S \Delta V}
$$

其中 $C_{\mathrm{S} 2}$ 为材料的质量比容量 $(\mathrm{F} / \mathrm{g}) ; I$ 为横流充放电 电流(A); $t$ 为放电时间( $(\mathrm{s}) ; m$ 为两电极的电极材料总 面积 $\left(\mathrm{cm}^{2}\right) ; V$ 为充放电去除欧姆电压降后的电压窗 口(V)。

\section{参考文献:}

[1] SONMEZ G, SHEN C K F, RUBIN Y, et al. A red, green, and blue (RGB) polymeric electrochromic device (PECD): the dawning of the PECD. Angew. Chem. Int. Ed., 2004, 43(12): 1498-1502.

[2] SHEN X, HE J, WANG N, et al. Graphdiyne for electrochemical energy storage devices. Acta Phys. -Chim. Sin., 2018, 34(9): 1029-1047. 